\title{
Kondo-type transport through an interacting quantum dot coupled to ferromagnetic leads
}

\author{
Bing Dong ${ }^{1,2}$, H. L. Cui ${ }^{1}$, S. Y. Liu $^{2}$, and X. L. Lei ${ }^{2}$ \\ ${ }^{1}$ Department of Physics and Engineering Physics, \\ Stevens Institute of Technology, Hoboken, New Jersey 07030 \\ ${ }^{2}$ Department of Physics, Shanghai Jiaotong University, 1954 Huashan Road, Shanghai 200030, China
}

\begin{abstract}
We investigate the equilibrium and out-of-equilibrium Kondo effects in a single-level interacting quantum dot connected to two ferromagnetic leads. Within the non-crossing approximation, we calculate the total density of states (DOS), the linear conductance, and the nonlinear differential conductance for both the parallel and the anti-parallel alignments of the spin polarization orientation in the leads, followed by a brief discussion regarding the validity of this approach. Numerical calculations show that for the anti-parallel alignment, a single Kondo peak always appears in the equilibrium DOS, resulting in the conventional temperature behavior in the linear conductance and the zero-bias maximum in the differential conductance. The strength of the DOS peak is gradually suppressed with increasing polarization, due to the fact that formation of the Kondocorrelated state is more difficult in the presence of higher polarization. On the contrary, for the parallel configuration the Kondo peak in the DOS descends precipitately and splits into two peaks to form a very steep valley between them. This splitting contributes to the appearance of a "hump" in the temperature-dependent linear conductance and a nonzero-bias maximum in the differential conductance. Moreover, application of a bias voltage can split each Kondo peak into two in the nonequilibrium DOS for both configurations. Finally we point out that the tunnel magnetoresistance could be an effective tool to demonstrate the different Kondo effects in different spin configurations found here.
\end{abstract}

PACS numbers: 72.10.Fk, 72.15.Qm, 72.25.Dc,73.63.Kv

\section{INTRODUCTION}

The discovery of the Kondo effect in a quantum dot (QD) connected to two normal reservoirs has stimulated much experimental and theoretical interests in this many-

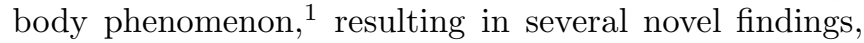
such as splitting of the Kondo peak under nonequilibrium condition,$\underline{2}$ an unususal enhancement of conductance in the cases when an even number of electrons resides in a $\mathrm{QD}, 3.4$ and double peaks of the differential conductance in coupled double QDs $\stackrel{5,6}{\cdot 6}$ Recently, an increasing attention has been paid, because of its potential application in the megnetoelectronics and quantum computer, ${ }^{7}$ to the spin-polarized electron tunneling through systems consisting of two ferromagnetic (FM) leads sandwiched by a QD, which features a significant Kondo effect at low temperatures when it connects to normal leads. Generally speaking, the Kondo resonance in the density of states (DOS) at the Fermi energy originates from screening of the dot spin due to the exchange coupling with the conduction electrons. Therefore it is interesting to observe whether the Kondo-correlated state can form when the conduction band is of spin polarization, and if so, what is the difference from the conventional patterns.

In a recent paper, Sergueev et al.$\frac{8}{n}$ and Zhang et $a l^{9} \underline{9}$ presented a theoretical analysis of the transport characteristics of such a FM/QD/FM system, using the ansatz proposed by $\mathrm{Ng}^{10}$ and the standard equation-ofmotion (EOM) technique for the retarded Green's function. They found that there is always a sharp single Kondo resonant peak in nonlinear differential conductiv- ity at zero bias, regardless of the polarization orientation of the two leads, parallel (P) or anti-parallel (AP) configurations. On the contrary, Martinek et al $\stackrel{11}{\underline{1}}$ reported a markedly different result on similar systems, based also on an EOM technique and an additional assumption to replace the bare level of QD in the resultant self-energy expression with the one self-consistenly determined. They found that for $\mathrm{P}$ alignment of the lead magnetizations, the Kondo resonances in the DOS split for spin-up and -down electrons, thus the differential conductance exhibits nonzero-bias maximum and the linear conductance drops to a low value even the polarization being as small as 0.2 . Later on, Lü and Liu $\frac{12}{2}$ reported also the similar splitting by applying Ng's ansatz. Ma et al $\underbrace{13} \mathrm{em}-$ ployed the finite- $U$ slave-boson mean-field approach ${ }^{14.15}$ to investigate the spin-polarized transport of this system and found drastically different behaviors depending on the polarization alignment of the two leads. In view of the ongoing controversy, further analysis on the influence of the spin polarization on the Kondo-correlated state in a QD is desirable, preferably based on more advanced schemes.

In this paper, we employ the non-crossing approximation (NCA) based on the auxiliary boson technique to carry out a detailed analysis of the FM/QD/FM Kondo problem. The NCA is a diagrammatic technique to sum all the non-crossing diagrams in the leading order $|V|^{2} / N$ ( $V$ is the hopping matrix element between the local electron and conduction electrons, and $N$ is the number of spin degeneracy of the local level) 16 And it is proved to be an accurate approach for the case of $N=2$ 
(of interest for a QD), even under out-of-equalibrium

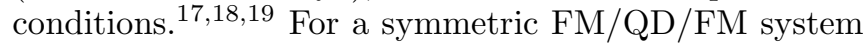
considered in the present paper, although the individual QD is exactly spin degenerate, the spin-polarized leads can lift this degeneracy in the case of $\mathrm{P}$ alignment through the tunneling induced spin-related self-energies. However, if the spin polarization strength $p$ of the two leads is small enough, the self-energies are of weak spindependence, and as a consequence the NCA should be adequate to treat this systems. Actually, we calculate the equilibrium and nonequilibrium transmissions for the FM/QD/FM system with the polarization strength as low as $p=0.2$ for the $\mathrm{P}$ configuration. Numerical results show a complete splitting for the equilibrium transmission (DOS) and a nonzero-bias maximum for the voltagedependent differential conductance, which are in qualitative agreement with those of Martinek ${ }^{11}$ and $\mathrm{Lü}^{12}$.

The rest of the paper is organized as follows. In section II we describe the model Hamiltonian and give a brief description of applications of the infinite- $U$ NCA to the spin-dependent transport in FM/QD/FM systems, as well as a qualitative discussion about its validity. In section III numerical computations and discussions are presented, including the equilibrium and out-of-equilibrium DOS, the linear conductance, and the nonlinear differential conductance, for both the $\mathrm{P}$ and the AP polarization configurations. Finally, all the results are summarized in Section IV.

\section{MODEL AND FORMULATION}

The system Hamiltonian for a quantum dot with a single spin-degenerate energy level $\epsilon_{d}(N=2)$ connected to two ferromagnetic leads is written as

$$
\begin{aligned}
H= & \sum_{\eta, k, \sigma} \epsilon_{\eta k \sigma} c_{\eta k \sigma}^{\dagger} c_{\eta k \sigma}+\epsilon_{d} \sum_{\sigma} c_{d \sigma}^{\dagger} c_{d \sigma} \\
& +U n_{d \uparrow} n_{d \downarrow}+\sum_{\eta, k, \sigma}\left(V_{\eta \sigma} c_{\eta k \sigma}^{\dagger} c_{d \sigma}+\text { H.c. }\right)
\end{aligned}
$$

where $c_{\eta k \sigma}^{\dagger}\left(c_{\eta k \sigma}\right)$ and $c_{d \sigma}^{\dagger}\left(c_{d \sigma}\right)$ are the creation (annihilation) operators for electrons with momentum $k$ and spin $\sigma$ in the lead $\eta(=\mathrm{L}, \mathrm{R})$ and for a spin- $\sigma$ electron on the $\mathrm{QD}$, respectively. The third term describes the Coulomb interaction among electrons on the QD, which is assumed to be infinite $(U \rightarrow \infty)$ in the present paper, forbidding double occupancy. The fourth term represents the tunneling coupling between the QD and the reservoirs via

$$
\Gamma_{\sigma}^{\eta}(\omega)=2 \pi \sum_{k}\left|V_{\eta \sigma}\right|^{2} \delta\left(\omega-\epsilon_{\eta k \sigma}\right), \quad(\eta=\mathrm{L}, \mathrm{R}) .
$$

In the wide band limit, $\Gamma_{\sigma}^{\eta}$ is assumed to be constant. For the identical leads and symmetric barriers, of interest in the present investigation, the ferromagnetism of the leads can be accounted for by the polarization-dependent couplings $\Gamma_{\uparrow}^{L}=\Gamma_{\downarrow}^{R}=(1+p) \Gamma_{0}, \Gamma_{\downarrow}^{L}=\Gamma_{\uparrow}^{R}=(1-p) \Gamma_{0}$ for the $\mathrm{P}$ alignment, while $\Gamma_{\uparrow}^{L}=\Gamma_{\downarrow}^{R}=(1+p) \Gamma_{0}, \Gamma_{\downarrow}^{L}=$ $\Gamma_{\uparrow}^{R}=(1-p) \Gamma_{0}$ for the AP alignment. $\Gamma_{0}$, and $p(0 \leq$ $p<1$ ) describe the tunneling coupling between the QD and the nonmagnetic leads, and the polarization strength of the leads. Under this approximation, the current $I$ through the QD can be expressed in terms of the total transmission $\mathcal{T}(\omega) \operatorname{as}^{20}$

$$
I=\frac{e}{\hbar} \int d \omega\left[f_{L}(\omega)-f_{R}(\omega)\right] \mathcal{T}(\omega),
$$

where

$$
\mathcal{T}(\omega)=\sum_{\sigma} \frac{\Gamma_{\sigma}^{L} \Gamma_{\sigma}^{R}}{\Gamma_{\sigma}^{L}+\Gamma_{\sigma}^{R}} \rho_{\sigma}(\omega)
$$

with $\rho_{\sigma}(\omega)=-\frac{1}{\pi} \operatorname{Im} G_{\sigma}^{r}(\omega)$ being the DOS for spin- $\sigma$ electrons. $G_{\sigma}^{r}(\omega)$ is Fourier transform of the retarded Green's function,

$$
G_{\sigma}^{r}(t)=-i \theta(t)\left\langle c_{\sigma}(t), c_{\sigma}^{\dagger}(0)\right\rangle .
$$

The main purpose of this work is to calculate the DOS $\rho_{\sigma}(\omega)$ as a function of temperature $T$, bare-level energy $\epsilon_{d}$ and bias voltage $V$ for different polarization configurations and strengths $p$, and the associated linear and nonlinear conductance.

According to the infinite- $U$ slave-boson approach, the ordinary electron operators on the QD can be decomposed into a boson operator $b$ and a pseudo-fermion operator $f_{\sigma}$,

$$
\begin{aligned}
& c_{d \sigma}(t)=b^{\dagger}(t) f_{\sigma}(t), \\
& c_{d \sigma}^{\dagger}(t)=f_{\sigma}^{\dagger}(t) b(t),
\end{aligned}
$$

with a constraint for the auxiliary operators $b^{\dagger} b+$ $\sum_{\sigma} f_{\sigma}^{\dagger} f_{\sigma}=1$. In the slave boson representation, the Hamiltonian (11) for the FM/QD/FM systems becomes

$$
\begin{aligned}
H= & \sum_{\eta, k, \sigma} \epsilon_{\eta k \sigma} c_{\eta k \sigma}^{\dagger} c_{\eta k \sigma}+\epsilon_{d} \sum_{\sigma} f_{\sigma}^{\dagger} f_{\sigma} \\
& +\sum_{\eta, k, \sigma}\left(V_{\eta \sigma} c_{\eta k \sigma}^{\dagger} b^{\dagger} f_{\sigma}+\text { H.c. }\right) .
\end{aligned}
$$

In order to evaluate the $\operatorname{DOS} \rho_{\sigma}(\omega)$, Wingreen et al ${ }^{18}$ generalized the NCA to study the nonequilibrium properties of the Anderson model connected with two normal conduction bands, using the Keldysh nonequilibrium Green's function formalism. It is well-known that the NCA is a self-consistent conserving perturbation expansion for the pseudo-fermion and slave-boson self-energies to first order in the effective coupling $J=|V|^{2}$. At the lowest order in perturbation diagrams the boson selfenergy involves the bare fermion propagator while the fermion self-energy involves the bare boson propagator. By replacing these bare propagators with the dressed auxiliary particle propagators in the Feynman diagram, one can obtain a set of coupled integral equations, which 
self-consistently determine the self-energies of these auxiliary particles. Solving these coupled equations is equivalent to summing up a subset of diagrams to all orders in $J$. Furthermore, it can be proved that the NCA includes all diagrams of leading orders in $1 / N, 16$ Therefore, the $\mathrm{NCA}$ is expected to be a quantitative approach in the limit of large $N$. For a QD connected with normal leads, $N=2$, it is already proved to be satisfactory in qualitatively describing the linear and nonlinear Kondo-type transport 18,19

Unfortunately, when the level degeneracy is broken, the NCA could produce spurious peaks in the DOS, thus is unreliable for transport investigation. For example, as mentioned by Wingreen, 18 the NCA without vertex corrections produces an additional Kondo peak at the chemical potential in a finite magnetic field due to a false sefl-interaction of each level, whereas other methods find that the Kondo peak splits into two peaks. Similarly, the NCA without vertex corrections seems to be also inappropriate for the FM/QD/FM systems, because spin-related tunneling lifts the level degeneracy in the QD. However, the present situation is somewhat different from magneto-transport. The degeneracy lifting is evident in the presence of a magnetic field, while is dependent on the relative polarization orientation of the two leads and of course the strength of polarization for the FM/QD/FM systems.

When the polarization orientations of the two FM leads is anti-parallel, the self-energies are actually independent of spin and the degeneracy remains as two for the case of the identical leads and symmetric barriers. On the other hand, for the $\mathrm{P}$ configuration spin-related tunneling results in the self-energies to be different for spin-up and -down electrons. However, it is natural that the deviation depends on the polarization strength $p$. Namely, the NCA could still be reliable for the FM/QD/FM systems with small enough $p$. Numerical calculations in the next section show that the conventional Kondo peak in DOS indeed splits into two peaks completely at the polarization $p=0.2$ for the $\mathrm{P}$ configuration. Even though one can observe an additional peak located at the chemical potential but an order of magnitude smaller than the two real Kondo peaks, these results are in agreement with previous predictions $\underline{11}$ Consequently, this convinces us that the NCA provides an appropriate description for the symmetric FM/QD/FM systems in either the AP alignment with arbitrary polarizations or the $\mathrm{P}$ configuration with weak polarizations. Of course, this scheme's validity being dependent on the polarization $p$ should be carefully checked, for example, by the modified NCA including vertex corrections. This, however, entails numerically a much heavier task than the original NCA and is beyond the purpose of the present paper. We leave this examination to a future publication.

We outline the formulation employed in this paper as follows. The interested reader can refer to Ref 18 and Ref 19 for detail. In the slave boson representation, the retarded Green's functions for the boson and pseudo- fermions are defined as

$$
\begin{aligned}
D^{r}(\omega) & =\frac{1}{\omega-\Pi^{r}(\omega)}, \\
G_{f \sigma}^{r}(\omega) & =\frac{1}{\omega-\epsilon_{d}-\Sigma_{f \sigma}^{r}(\omega)},
\end{aligned}
$$

with the corresponding retarded self-energies $\Pi^{r}(\omega)$ and $\Sigma_{f \sigma}^{r}(\omega)$. Furthermore, the "lesser" Green's functions for the boson and fermions are related with the "lesser" selfenergies $\Pi^{<}(\omega)$ and $\Sigma_{f \sigma}^{<}(\omega)$ as

$$
\begin{aligned}
D^{<}(\omega) & =D^{r}(\omega) \Pi^{<}(\omega) D^{a}(\omega), \\
G_{f \sigma}^{<}(\omega) & =G_{f \sigma}^{<}(\omega) \Sigma_{f \sigma}^{<}(\omega) G_{f \sigma}^{a}(\omega) .
\end{aligned}
$$

The self-consistent NCA equations for out-of-equilibrium are

$$
\begin{aligned}
\Pi^{r}(\omega) & =\sum_{\eta=\mathrm{L}, \mathrm{R}, \sigma} \frac{\Gamma_{\sigma}^{\eta}}{2 \pi} \int d \varepsilon f\left(\varepsilon-\omega-\mu_{\eta}\right) G_{f \sigma}^{r}(\varepsilon), \\
\Sigma_{f \sigma}^{r}(\omega) & =\sum_{\eta=\mathrm{L}, \mathrm{R}} \frac{\Gamma_{\sigma}^{\eta}}{2 \pi} \int d \varepsilon f\left(\varepsilon-\omega+\mu_{\eta}\right) D^{r}(\varepsilon), \\
\Pi^{<}(\omega) & =\sum_{\eta=\mathrm{L}, \mathrm{R}, \sigma} \frac{\Gamma_{\sigma}^{\eta}}{2 \pi} \int d \varepsilon f\left(\varepsilon-\omega+\mu_{\eta}\right) G_{f \sigma}^{<}(\varepsilon), \\
\Sigma_{f \sigma}^{<}(\omega) & =\sum_{\eta=\mathrm{L}, \mathrm{R}} \frac{\Gamma_{\sigma}^{\eta}}{2 \pi} \int d \varepsilon f\left(\varepsilon-\omega-\mu_{\eta}\right) D^{<}(\varepsilon),
\end{aligned}
$$

where $f(x)=[\exp (\beta x)+1]^{-1}\left(\beta=1 / k_{\mathrm{B}} T\right)$ is the Fermi distribution function and $\mu_{\eta}$ is the chemical potential of the $\eta$ lead. After solving this set of self-consistent equations, the imaginary part of the retarded local Green's function, the DOS $\rho_{\sigma}(\omega)$, can be calculated within the NCA as

$$
\begin{aligned}
\rho_{\sigma}(\omega) & =\frac{1}{Z} \int \frac{d \varepsilon}{2 \pi}\left[D^{<}(\varepsilon) \operatorname{Im} G_{f \sigma}^{r}(\varepsilon+\omega)\right. \\
& \left.+G_{f \sigma}^{<}(\varepsilon) \operatorname{Im} D^{r}(\varepsilon-\omega)\right],
\end{aligned}
$$

where

$$
Z=\int d \varepsilon\left[D^{<}(\varepsilon)+\sum_{\sigma} G_{f \sigma}^{<}(\varepsilon)\right]
$$

Finally we can use Eqs.(3) and (4) to calculate the current through the QD.

\section{NUMERICAL RESULTS AND DISCUSSIONS}

\section{A. Density of State}

In this section we present numerical calculations and discussions. First, we deal with the total equilibrium and out-of-equilibrium DOS (transmission) Eq. (4) for the FM/QD/FM systems with a fixed bare-level energy $\epsilon_{d}=-4.0\left(\Gamma_{0}\right.$ is used as the energy unit throughout the 
rest of the paper). It is worth noting that the systems considered here belong to the deep Kondo regime and are appropriate to demonstrate the strong correlated effects.

Fig. 1(a) shows the total DOS in the AP configuration for several different polarizations $p=0,0.2$, and 0.4 as well as various temperatures $T=0.01,0.02$, and 0.04 . Clearly, a significant Kondo peak remains at the Fermi energy (which is chosen to be the the energy zero) under the addition of the spin-polarized leads. The contribution of spin-polarized leads is to suppress both the Kondo peak and the single particle excitation peak (see overall shapes of the DOS in the inset of Fig. 1). This suppression is more pronounced with increasing polarization $p$. These results are understandable with the aid of the following considerations. Suppose that in the extreme case of two completely spin-polarized leads $p=1$, spin-down electrons are completely absent in the left lead, but electrons in the right lead are all spin-up and could provide compensation to screen the dot spin and to guarantee the formation of the Kondo-correlated singlet state. Thus the Kondo peak still exists with a reduced amplitude. Naturally, the cases of weak polarizations $p<1$ are more likely to form the Kondo state. Note that increasing temperature can broaden the peak and suppress the Kondo resonance as usual. In addition, effects of the external bias voltage on the out-of-equilibrium DOS are plotted in Fig. 1(b). For convenience, we choose a symmetric voltage drop such that the chemical potential $\mu_{L}=-\mu_{R}=e V / 2$ for the left and right leads. As expected, we find a splitting of the Kondo peak with a width nearly equal to the bias voltage applied between the source and drain leads.

In short, the QD connected with two AP magnetized leads develops the same Kondo resonance as the QD with two normal leads, whereas the former is suppressed to some extent depending on the polarization $p$. In contrast, the situation is drastically different for the $\mathrm{P}$ configuration as shown in Fig. 2, where we plot the total DOS for the polarization $p=0.2$ with various temperatures (a) and bias voltages (b). It is clear that the $\mathrm{P}$ polarization significantly changes the DOS of the QD in comparison with the case of $p=0$. The Kondo resonance splits into two distinct peaks with different amplitudes. One moves from the original location $\omega=0$, the Fermi energies of the two leads, to a lower energy position, the other shifts to the opposite direction at the expense of its height. Moreover the magnitudes of both Kondo resonances are largely suppressed by the introduction of spin polarization. We observe that there appears a remnant peak located at the Fermi energy, which is produced by the NCA calculation without vertex corrections as mentioned above. But its amplitude is one order smaller than the two shifted Kondo peaks and can be neglected. As a result, a very steep valley is found between the two peaks with a nearly vanished bottom. Finally we find that increasing temperature can not only smooth and broaden the peaks as usual, but also raise the bottom of the deep valley gradually, which can result in a peculiar temper- ature dependence of the linear conductance as shown in Fig. 3 (in the next subsection).

Fig. 2(b) depicts effects of changing bias voltage on the total DOS (transmission probability) for the same system as in Fig. 2(a). If we keep the temperature low, $T=0.01$, and increase the bias voltage, the two resonances first experience suppression and then each of them splits into two distinct peaks. Each pair of the peaks has a width about equal to the bias voltage. Increasing the temperature would eventually wash out the peak splitting and recover a single but much less pronounced peak in both equilibrium and out-of-equilibrium cases.

\section{B. Linear and Nonlinear Conductance}

In Fig. 3 we plot the calculated linear response conductance $G$ vs. $\log T$ for various polarizations $p=$ $0,0.1,0.15$, and 0.2 . In the AP configuration (thin line in Fig. 3) the linear conductance $G_{A P}$ exhibits the similar overall temperature dependence with those of nonmagnetic leads $p=0$, though smaller in magnitude resulted from the suppression of the Kondo resonance as addressed in Fig. 1(a). For the opposite orientation, the conductance $G_{P}$ depends strongly on the polarization strength $p$ and exhibits no universal $T$ behavior at low temperatures. Increasing $p, G_{P}$ is largely suppressed first and develops a "hump" as a function of temperature. This peak is due to the fact that the Kondo resonance is shifted away from the Fermi energy as shown in Fig. 2(a). To demonstrate the dramatic change of conductance under different polarization orientations, we plot the tunnel magnetoresistance (TMR) defined as $T M R=\left(G_{P}-G_{A P}\right) / G_{A P}$ in the inset of Fig. 3. We find the TMR arrives at a value as large as $100 \%$ at the lowest temperature calculated in this work and falls rapidly with increasing $T$, which can be attributed to the peculiar Kondo resonance in the $\mathrm{P}$ configuration. At high temperatures $G_{P}$ approaches the value of nonpolarization $p=0$, leading to a saturated and small positive linear TMR.

Fig. 4 shows the linear conductance as a function of bare-level energy $\epsilon_{d}$, which can be tuned via the external gate voltage, for nonpolarization $p=0$, as well as the $\mathrm{P}$ and the AP configurations with $p=0.2$. As expected, $G_{A P}$ shows the same trend with that of nonpolarization though a smaller amplitude. The peak of $G_{p}$, however, shifts towards the Fermi energy. This means that the linear TMR changes its sign at a certain level energy and has approximatively a symmetric shape around this point. Near the empty orbital regime $\epsilon_{d} \simeq 0$ and near the deep Kondo regime $\epsilon_{d} \simeq-4$, the linear TMR reaches its maximum value as large as $30 \%$ in the temperature $T=0.1$.

Nonlinear differential conductance $d I / d V$ is believed to be a very useful and sensitive tool in experiments to detect the formation of the Kondo-correlated state due to its proportionality to $\mathcal{T}(e V)$ derived from the current for- 
mula Eq.(3), assuming that the total transmission $\mathcal{T}(\omega)$ (nonequilibrium DOS) is unchanged under the external bias voltage $V$. So we illustrate in Fig. 5 the calculated $d I / d V$ under the $\mathrm{P}(\mathrm{a})$ and the $\mathrm{AP}(\mathrm{b})$ configurations, as well as the nonlinear TMR (c) at various temperatures. As pointed out above, because electrons with spin-up and spin-down are equally available in the AP configuration, the formation of the Kondo-correlated state should not be affected. As a result, all the curves in Fig. 5(b) exhibit a single zero-bias peak and rapid decrease in peak height with increasing temperature. When the magnetization is rotated to the $\mathrm{P}$ alignment, adding bias voltage can greatly enhance $d I / d V$ at low temperatures. This is apparently due to the complete splitting of the Kondo peak in the DOS shown in Fig. 2. The nonzero-bias maximum in $d I / d V$ is in good agreement with previous EOM

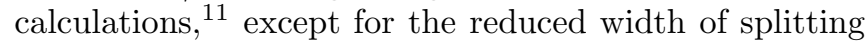
and the fine shape in the differential conductance. These inconsistences can be attributed to the significant change of the nonequilibrium DOS with increasing bias voltage [see Fig. 2(b)]. Furthermore, the nonlinear TMR displays a deep dip in the linear regime and changes its sign at a certain bias voltage. This large change in the TMR vs. bias voltage reflects the different behaviors of the Kondo resonance in the $\mathrm{P}$ and the $\mathrm{AP}$ configurations.

\section{SUMMARY}

We have investigated the low-temperature, nonequilibrium properties of a spin-valve system consisting of a QD connected to two ferromagnetic leads in the Kondo regime. Based on the NCA approach we find markedly different behaviors in the equilibrium DOS when changing the relative orientation of spin polarization. In the AP configuration, we find that a single Kondo peak always appears through the whole range of polarization $0 \leq p \leq 1$, as just in a $\mathrm{QD}$ connected to two normal leads. Increasing polarization $p$ can slightly suppress the amplitude of the peak. In the $\mathrm{P}$ configuration, the Kondo peak descends greatly and splits completely into two peaks even for a weak polarization as low as $p=0.2$, leading to a steep valley with nearly a zero bottom. In both configurations the chemical-potential difference (the bias voltage) appears in the DOS via the the splitting of the Kondo peak into two peaks. Thus four peaks can be found in a moderate bias voltage for the $\mathrm{P}$ alignment. Of course the amplitudes of these peaks are suppressed by increasing temperature.

Experimentally, we predict, based on the NCA investigation, that the different Kondo effects can be observed in transport through a QD by either linear or nonlinear measurements. For the AP configuration, the calculations exhibit the usual temperature dependence of the linear conductance and a zero-bias maximum in the nonlinear conductance, which are the conventional properties of the Kondo-dominated transport through a QD. For the P configuration, however, we find a "hump" in the temperature-dependent linear conductance and a nonzero-bias maximum in the differential conductance. These peculiar behaviors are associated with the fact that the Kondo peak of the QD is split and shifted away from the Fermi level in the case of the $\mathrm{P}$ alignment. Furthermore, we suggest that the TMR is a more effective tool to explore the different features of the Kondo resonance in different configurations.

Finally, we point out again the applicability of the NCA approach to the FM/QD/FM systems. No spin splitting in the case of the AP alignment guarantees that the NCA is a reliable approximation for quantities involving the DOS. For the $\mathrm{P}$ configuration our numerical results for the DOS show satisfactory agreement with previous EOM predictions in the case of weak polarization $p=0.2$. Thus we believe that the self-consistent second-order perturbation approach provides some qualitative features of the Kondo effect in the DOS as long as the polarizations of the leads are weak enough, which can serve to furnish a deeper understanding of transport properties in FM/QD/FM systems. Advanced NCA studies containing the vertex corrections are required to examine the validity of this approach. Work along this line is in progress.

\section{Acknowledgments}

B. Dong and H. L. Cui are supported by the DURINT Program administered by the US Army Research Office. S. Y. Liu and X. L. Lei are supported by the National Science Foundation of China, the Special Funds for Major State Basic Research Project, and the Shanghai Municipal Commission of Science and Technology.
1 D. Goldhaber-Gordon, H. Shtrikman, D. Mahalu, D. Abusch-Magder, U. Meirav and M. A. Kastner, Nature (London) 391, 156 (1998); S. M. Cronenwett, T. H. Oosterkamp, and L. P. Kouwenhoven, Science 281, 540 (1998); W. G. van der Wiel, S. De Franceschi, T. Fujisawa, J. M. Elzerman, S. Tarucha and L. P. Kouwenhoven, Science 289, 2105 (2000).

2 J. Schmid, J. Weis, K. Eberl and K. v. Klitzing, Phys. Rev. Lett. 83, 5824 (2000).
${ }^{3}$ S. Sasaki, S. De Franceschi, J. M. Elzerman, W. G. van der Wiel, M. Eto, S. Tarucha, and L. P. Kouwenhoven, Nature 405, 764 (2000); J. Nygård, D. H. Cobden and P. E. Lindelof, Nature 408, 342 (2000).

${ }^{4}$ W. G. van der Wiel, S. De Franceschi, J. M. Elzerman, S. Tarucha and L. P. Kouwenhoven, J. Motohisa, F. Nakajima and T. Fukui, Phys. Rev. Lett. 88, 126803 (2002).

5 T. Fujisawa, et al, Science 282, 932 (1998); T. H. Oosterkamp, et al, Nature 395, 873 (1998). 
${ }^{6}$ H. Jeong, A. M. Chang, M. R. Melloch, Science 293, 2221 (2001).

7 G. A. Prinz, Science 282, 1660 (1998).

8 N. Sergueev, Qing-feng Sun, Hong Guo, B. G. Wang and Jian Wang, Phys. Rev. B 65, 165303 (2002).

9 P. Zhang, Q. K. Xue, Y. P. Wang, and X. C. Xie, Phys. Rev. Lett. 89, 286803 (2002).

10 T. K. Ng, Phys. Rev. Lett. 70, 3635 (1993).

11 J. Martinek, Y. Utsumi, H. Imamura, J. Barnaś, S. Maekawa, J. König, and G. Schön, cond-mat/0210006 (2002).

12 R. Lü and Z.R. Liu, cond-mat/0210350 (2002).

13 Jing Ma, Bing Dong, and X. L. Lei, cond-mat/0212645 (2002).

14 G. Kotliar and A. E. Ruckenstein, Phys. Rev. Lett. 57, 1362 (1986).

15 Bing Dong and X. L. Lei, J. Phys: Condens. Matter 13, 9245 (2001); Phys. Rev. B 63, 235306 (2001); Phys. Rev. B 65, 241304 (2002).

16 N. E. Bickers, Rev. Mod. Phys. 59, 845 (1987).

17 D. L. Cox, Phys. Rev. B 35, 4561 (1987).

18 Ned S. Wingreen, and Y. Meir, Phys. Rev. B 49, 11040 (1994).

19 M. H. Hettler, J. Kroha, and S. Hershfield, Phys. Rev. B 88, 5649 (1998).

20 Y. Meir and N. S. Wingreen, Phys. Rev. Lett. 68, 2512 (1992).

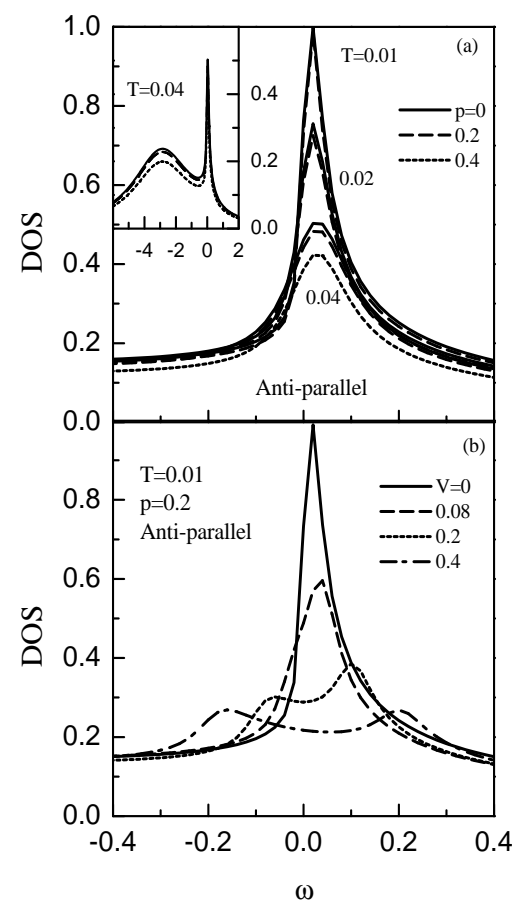

FIG. 1: The total equilibrium DOS $\mathcal{T}(\omega)$ in the AP configuration for different temperatures $T / \Gamma_{0}=0.01,0.02$, and 0.04 and different polarizations $p=0,0.2$ and 0.4 ; and (b) the nonequilibrium DOS for $T=0.01$ and $p=0.2$. The QD in the $\mathrm{FM} / \mathrm{QD} / \mathrm{FM}$ system has a single bare-level energy $\epsilon_{d}=-4.0$ and an infinite on-site Coulomb interaction $U \rightarrow \infty$. 


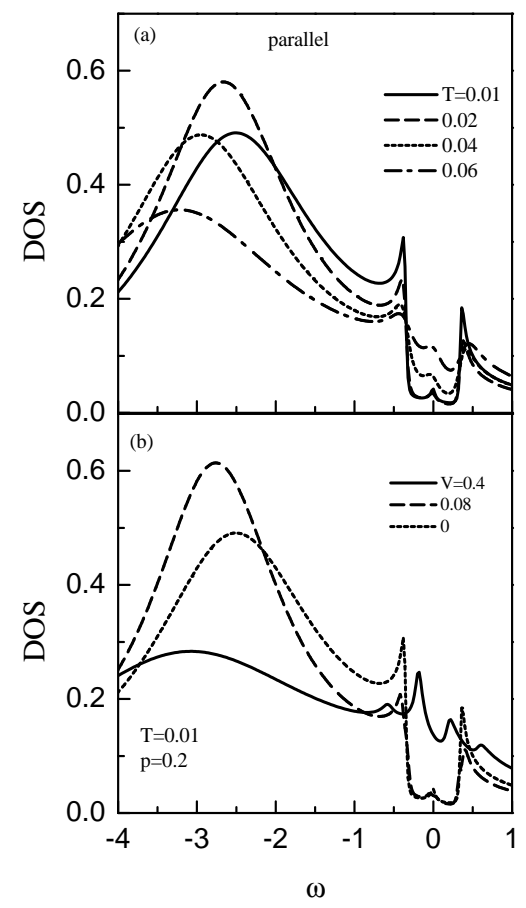

FIG. 2: (a) The total equilibrium DOS in the $\mathrm{P}$ configuration for different temperatures $T / \Gamma_{0}=0.01,0.02,0.04$, and 0.06 ; and (b) the nonequilibrium DOS for $T=0.01$ and $p=0.2$. The system is the same as described in Fig. 1.

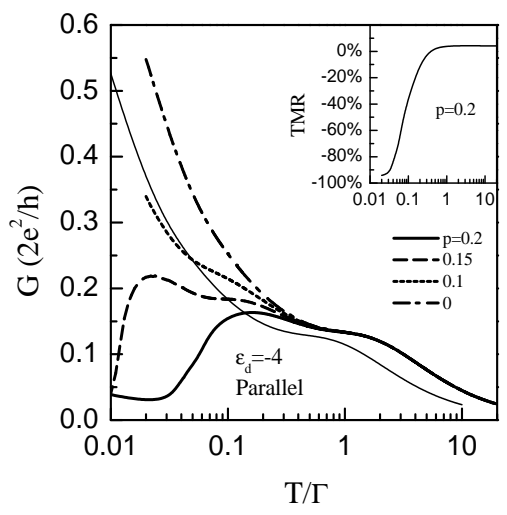

FIG. 3: The linear conductance $G$ versus temperature at different polarizations $p$ for the same system as described in Fig. 1. The thick lines correspond to the results for the $\mathrm{P}$ alignment, while the thin curve for the AP alignment at $p=0.2$. Inset: The calculated TMR vs. temperature for $p=0.2$ 


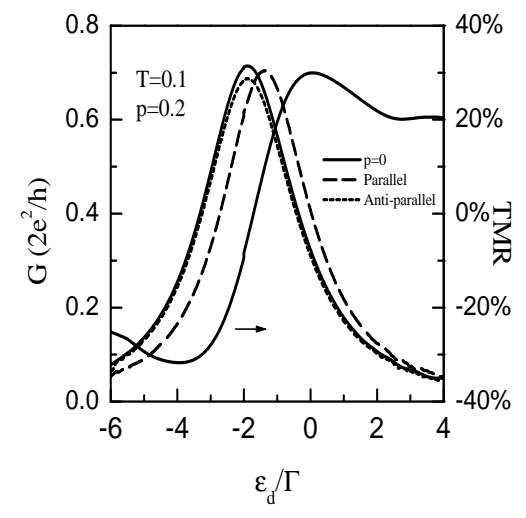

FIG. 4: The linear conductance $G$ and the TMR versus the bare-level energy of the QD at $T=0.1$.

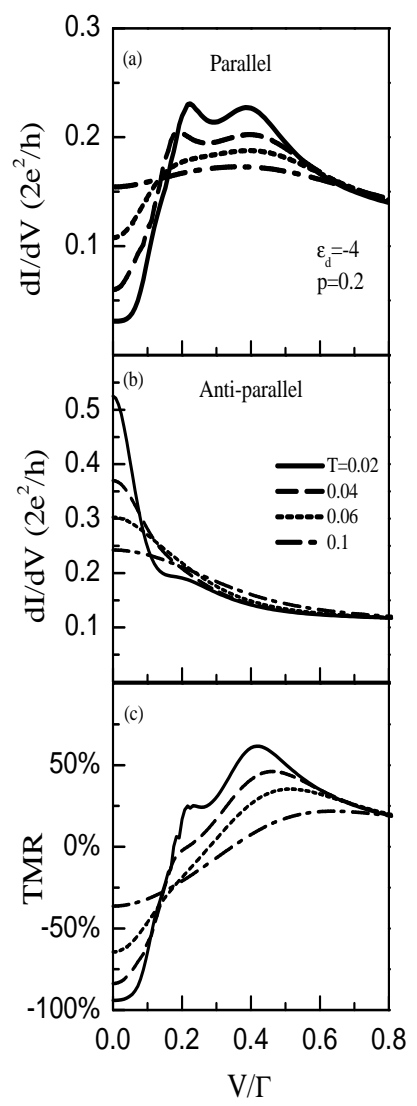

FIG. 5: The differential conductance $d I / d V$ versus the bias voltage $e V$ at different temperatures $T=0.02,0.04,0.06$, and 0.1 in the $\mathrm{P}$ (a) and the AP (b) configurations. (c) The nonlinear TMR versus the bias voltage. 